\title{
Salmonella grows massively and aerobically in fecal matter
}

\author{
Teresa Guerrero ${ }^{1}$, Sonia Zapata ${ }^{1}$ and Gabriel Trueba ${ }^{1}$
}

1 Microbiology Institute, Universidad San Francisco de Quito, Diego de Robles y Vía Interoceánica, Cumbayá, Quito, Ecuador.

\section{Summary}

The use of wastewater for irrigation and animal manure as fertilizer can cause transmission of intestinal pathogens, conditions frequently observed in Low- and Middle-Income Countries (LMICs). Here we tested the ability of Salmonella to grow in the fecal matter; we inoculated freshly isolated Salmonella strains (from chickens) in chicken fecal matter and incubated for 24, 48 and $72 \mathrm{hrs}$ under aerobic and anaerobic conditions. We found that both Salmonella and E. coli multiplied massively in fecal matter outside a host for $72 \mathrm{hrs}$, being their growth higher in aerobic conditions. Our results have critical implications in waste management, as we demonstrate that aerobic treatments may not be the best to reduce the number of Salmonella in the environment.

\section{Introduction}

Environmental transmission of intestinal pathogens is extremely important especially in Lowand Middle-Income Countries (LMICS) due to deficient sanitary infrastructure, unplanned urban growth, lack of wastewater treatment, etc. One of the main concerns in LMICs is the large proportion of untreated wastewater used for irrigation (Khalid et al., 2018) and the increasing use of animal manure as fertilizer without suitable treatment (Mandrell, 2009); these are problems that remain neglected in LMICs (Khalid et al., 2018). Reports of grave enteric infections caused by environmental contamination of produce are also commonplace nowadays in industrialized countries (Callejón et al., 2015). Some of these outbreaks have been associated with high mortality, morbidity and large economic losses (Mandrell, 2009). 
The incidence of these infections is exacerbated by the increasing appeal to consume natural, non-processed fresh products (Mandrell, 2009).

Salmonella contaminated water is responsible for a large number of outbreaks by ingestion of water or produce (Mandrell, 2009); the sources for this contamination are human and nonhuman fecal matter (Medrano-Félix et al., 2017). The use of animal waste as fertilizer constitutes a serious risk which can be controlled by appropriate composting technology (Tiquia et al., 1998; Szogi et al., 2015). Human waste contamination, however, is much more difficult to monitor or control in LMICs where wastewater treatment or toilets are not available (Khalid et al., 2018); the fate of Salmonella in these conditions is not understood completely, although some researchers indicate that Salmonella enters into a viable-non culturable state outside the host (Winfield and Groisman, 2003). The reduction of the risk of this type of transmission requires the understanding of every aspect of Salmonella physiology in the environment outside the host (Mandrell, 2009). It is worth mentioning that Salmonella's ability to grow in fecal matter has been ignored.

It is known that Salmonella and other Enterobacteriaceae survive in a fecal matter for some time and it has been shown that E. coli (another member of the Enterobacteriaceae) also grows massively in fecal matter (Russell and Jarvis, 2001; Vasco et al., 2015; Sharma et al., 2019). Here we tested Salmonella's ability to grow in fecal matter in aerobic conditions and discuss the potential implications for fecal waste management.

\section{Experimental Procedures}

\section{Overall approach}

We inoculated fresh Salmonella isolates in chicken fecal matter and incubated for 24,48 and $72 \mathrm{hrs}$ under aerobic and anaerobic conditions; we counted Salmonella colonies number by culture and by the loop mediated isothermal amplification $3 \mathrm{M}^{\mathrm{TM}}$ Molecular Detection Assay 2 Salmonella (MDA2SAL). 
Five fresh Salmonella isolates, obtained from poultry, were identified as S. enterica serovars Infantis, Dublin, Heidelberg, Brandenburg and Stanley by means of a multiplex PCR (Kim et al., 2006). A S. Infantis resistant to nitrofurantoin was used for plate count tests.

Salmonella inoculation in chicken fecal matter

Each Salmonella strain was cultured in $4 \mathrm{~mL}$ of $\mathrm{BHI}$, then the culture was centrifuged for $5 \mathrm{~min}$ at 4,000 $\mathrm{xg}$; the supernatant was discarded, and the pellet was re-suspended in $500 \mu \mathrm{L}$ of sterile saline solution. The process was repeated once to eliminate remnants of culture medium, and the resulting suspension was used to inoculate chicken feces. Fecal material was obtained from 3 2-week old broiler chickens free of Salmonella.

Fecal matter from the 3 chickens was pooled and split into seven 10 gram aliquots, placed in seven Petri dishes, mixed with $100 \mu \mathrm{L}$ of each Salmonella strains, and three dishes were left in an aerobic environment and three in anaerobic conditions; all dishes were placed at room temperature and incubated for 24,48 and 72 hrs (Vasco et al., 2015). Anaerobic atmosphere was created by means of BD GasPak ${ }^{\mathrm{TM}}$ EZ Anaerobe Gas Generating Pouch System with Indicator.

Colony counts

Two dishes containing fecal matter with S. Infantis (strain POL 398 B) suspension (1.5 $\times 10^{9}$ cells per $\mathrm{mL}$ ) incubated as before under aerobic and anaerobic conditions was subjected to colony count in culture media for Salmonella, E. coli and coliforms ( 0 hrs). After incubation, contents of the different dishes were diluted. Dilutions were made up to $10^{-8}$ in buffered peptone water (BPW), and the dilutions $10^{-6}, 10^{-7}$ and $10^{-8}$ were inoculated using plate pour method onto XLD and XLD with nitrofurantoin (NIT) $(12 \mathrm{mg} / \mathrm{L}$ ) (Sandegren et al., 2008) (we took advantage of the Salmonella strain's resistance to nitrofurantoin to facilitate Salmonella colony count). Typical Salmonella colonies were counted in XLD and XLD with NIT. The three mentioned dilutions from each one of the fecal matters (aerobic or anaerobic) dishes were 
inoculated onto $3 \mathrm{M}^{\mathrm{TM}}$ Petrifilm E. coli/Coliform Count Plates (in duplicate) and incubated for 24 and $48 \mathrm{hrs}$ at $37^{\circ} \mathrm{C}$. We counted red colonies (E. coli) from dishes incubated 24 and $48 \mathrm{hrs}$ (from both aerobic and anaerobic). The number of coliforms corresponded to the sum of the red and blue colonies. For each treatment, we included and analyzed a fecal sample without Salmonella inoculation as a control.

Calculation of specific growth rate, $\mu$

The specific growth rate $(\mu)$ was calculated using the formula:

$$
\mu=\frac{2.3 \log \left(\frac{\mathrm{N}}{\mathrm{No}_{\mathrm{O}}}\right)}{\Delta \mathrm{t}}
$$

where $\mathrm{N}$ is the final population after a time interval of incubation, $\Delta \mathrm{t}$, and No is the initial population (Maier, 2009; Montville et al., 2012).

\section{Quantitative PCR}

We determine the increase in Salmonella cells in chicken fecal matter at different time points by the loop mediated isothermal amplification method 3M $\mathrm{M}^{\mathrm{TM}}$ Molecular Detection Assay 2 Salmonella (MDA2SAL). For this purpose, we performed a calibration curve (Gandelman et al., 2010) as follows. We prepared a stool suspension with $1 \mathrm{~g}$ of chicken fecal matter and $9 \mathrm{~mL}$ of BPW (stool dilution). For the $10^{-1}$ standard suspension we added to $900 \mu \mathrm{L}$ of the stool dilution $100 \mu \mathrm{L}$ of a Salmonella Infantis (strain POL 398 B) suspension whose cell concentration was determined by counting in a Petroff-Hausser chamber. For each of the following standard suspensions $\left(10^{-2}\right.$ to $\left.10^{-10}\right)$, we used $900 \mu \mathrm{L}$ of stool dilution and $100 \mu \mathrm{L}$ of successive dilutions of the $S$. Infantis suspension. Then we mix $20 \mu \mathrm{L}$ of each standard suspension with the reagents of the kit 3M ${ }^{\mathrm{TM}}$ MDA2SAL and analyze them following manufacturer instructions.

Quantitative PCR was carried out after different incubation times (0, 24, 48 and $72 \mathrm{hrs})$; we made a $10^{-1}$ dilution of the chicken feces (inoculated with each serovar: Infantis, Dublin, Heidelberg, Brandenburg and Stanley) and analyzed using 3M ${ }^{\mathrm{TM}}$ MDA2SAL. We used regression 
equation to calculate the concentration of Salmonella cells at each time point (Gandelman et al., 2010). Additionally, for each trial we run suspensions of the strains analyzed, whose concentration (number of cells per $\mathrm{mL}$ ) was determined in the Petroff-Hausser chamber.

\section{Statistic analysis}

Using SPSS, we performed the U Mann-Whitney test, which compares independent samples that do not have a normal distribution (it is the non-parametric version of the Student's t-test).

\section{Results and discussion}

We found that Salmonella Infantis inoculated in chicken fecal matter multiplied, both in aerobic and anaerobic conditions, however, the aerobic growth was higher, notably from 48 to $72 \mathrm{hrs}$. Conversely, E. coli growth reached its peak after $48 \mathrm{hrs,} \mathrm{from} \mathrm{then} \mathrm{on,} \mathrm{the} \mathrm{growth}$ decreased (Fig. 1). Coliforms grew similarly but their numbers were higher (Supporting Information Fig. S1).

\section{$2.41 \mathrm{E}+08$}

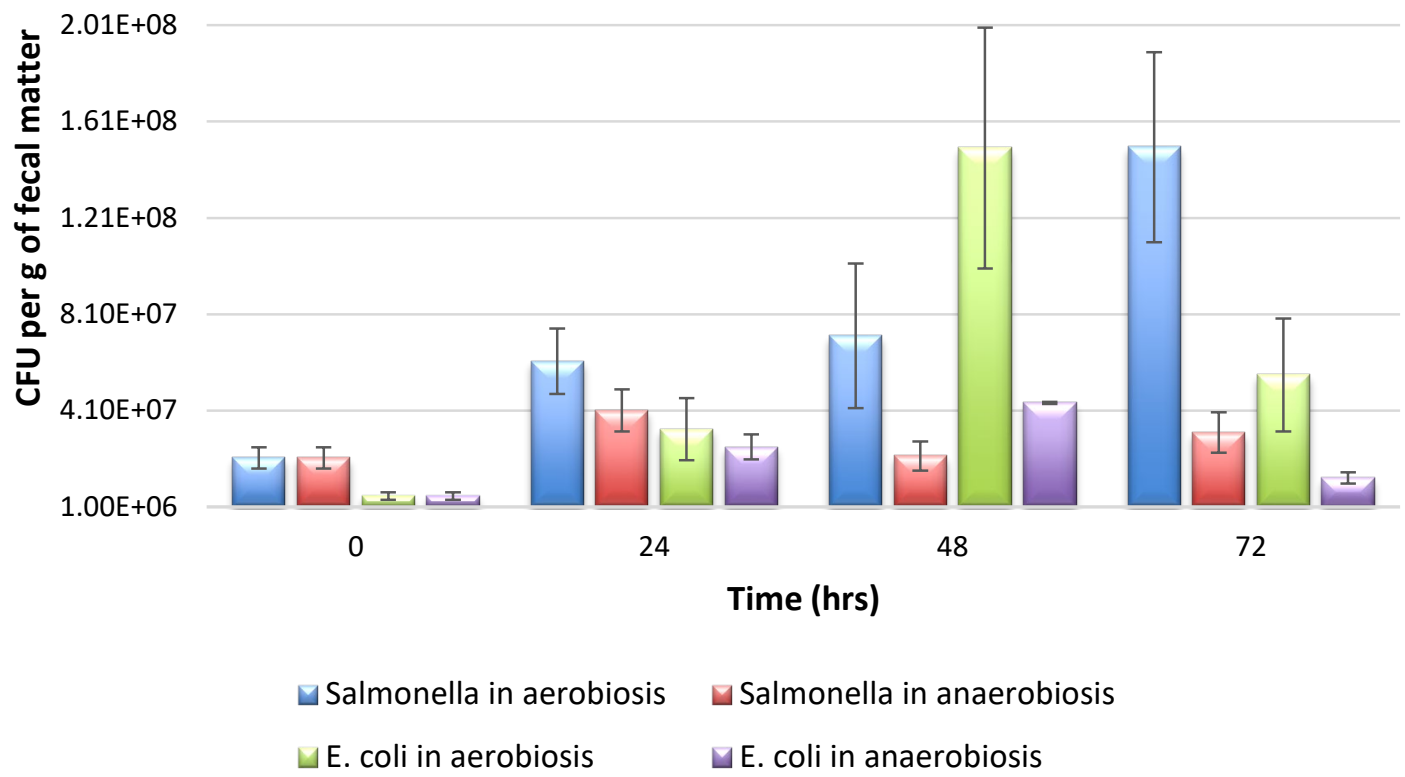

Fig. 1. Growth of Salmonella Infantis and $E$. coli in chicken fecal matter, under aerobic and anaerobic conditions. Typical Salmonella colonies were counted in XLD and XLD with NIT (12 $\mathrm{mg} / \mathrm{L}$ ) (we took advantage of the Salmonella strain's resistance to nitrofurantoin to facilitate Salmonella colony count). E. coli was counted in $3 \mathrm{M}^{\mathrm{TM}}$ Petrifilm E. coli/Coliform Count Plates (in duplicate). Bars indicate standard error. 
bioRxiv preprint doi: https://doi.org/10 1101/766782; this version posted September 12 2019. The copyright holder has placed this preprint (which was not certified by peer review) in the Public Domain. It is no longer restricted by copyright. Anyone can legally share, reuse, remix, or adapt this material for any purpose without crediting the original authors.

To determine whether this aerobic growth pattern could be extrapolated to other Salmonella serovars we inoculated chicken fecal matter with Salmonella strains (belonging to 5 different serovars), run the loop mediated isothermal amplification 3M $\mathrm{M}^{\mathrm{TM}}$ Molecular Detection Assay 2 Salmonella (MDA2SAL) at different times (under aerobiosis and anaerobiosis), and found that all strains showed similar behavior (Fig. 2).

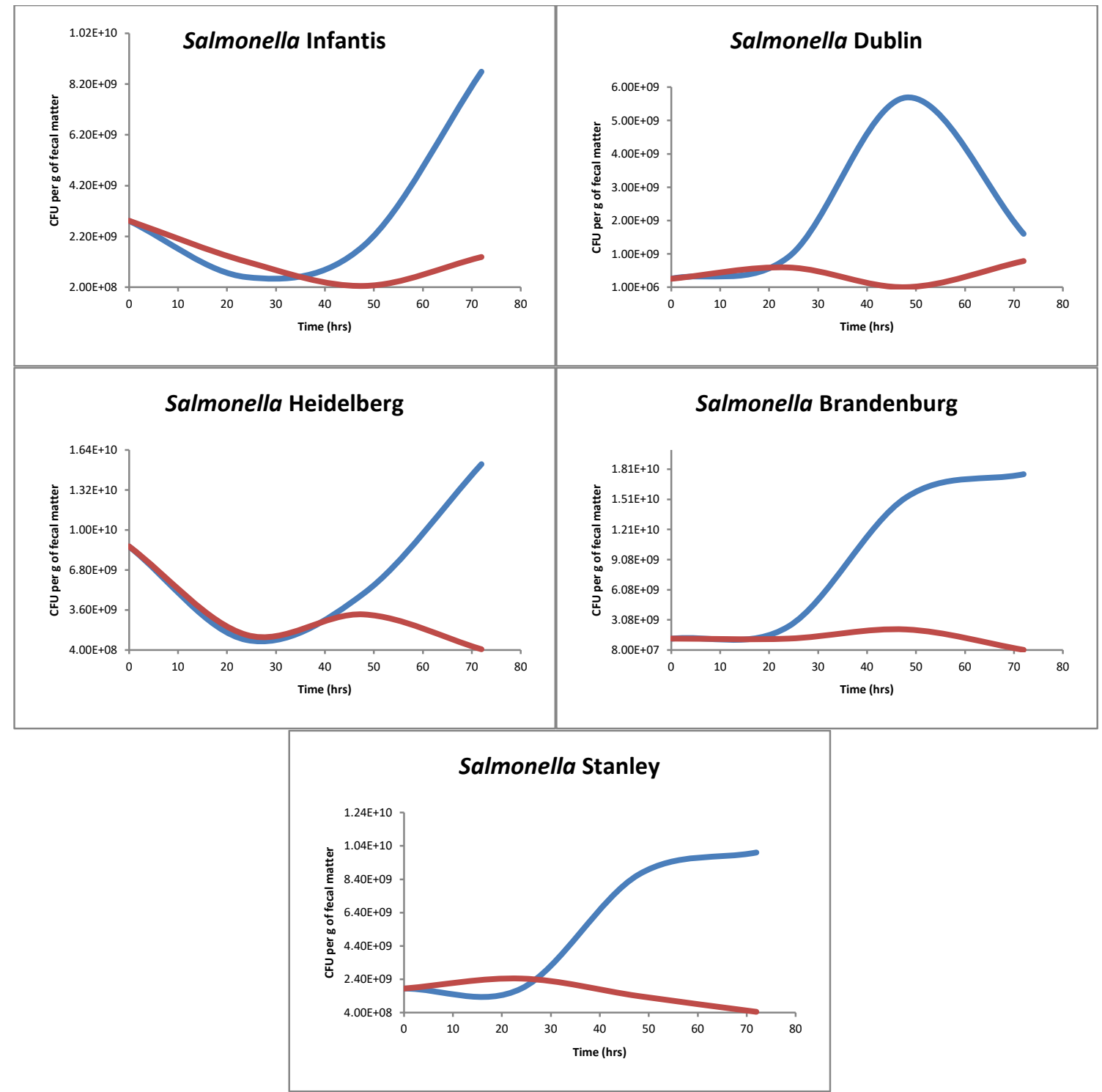

Fig. 2. Growth curves of five Salmonella serovars. These curves were obtained for five Salmonella serovars by $3 \mathrm{M}^{\mathrm{TM}}$ Molecular Detection Assay 2 - Salmonella (MDA2SAL). The blue lines correspond to the growth under aerobic conditions and red ones, to the growth under anaerobic conditions.

Salmonella spp. growth under aerobic conditions continued for $72 \mathrm{hrs} \mathrm{(Fig.} \mathrm{3).}$ 


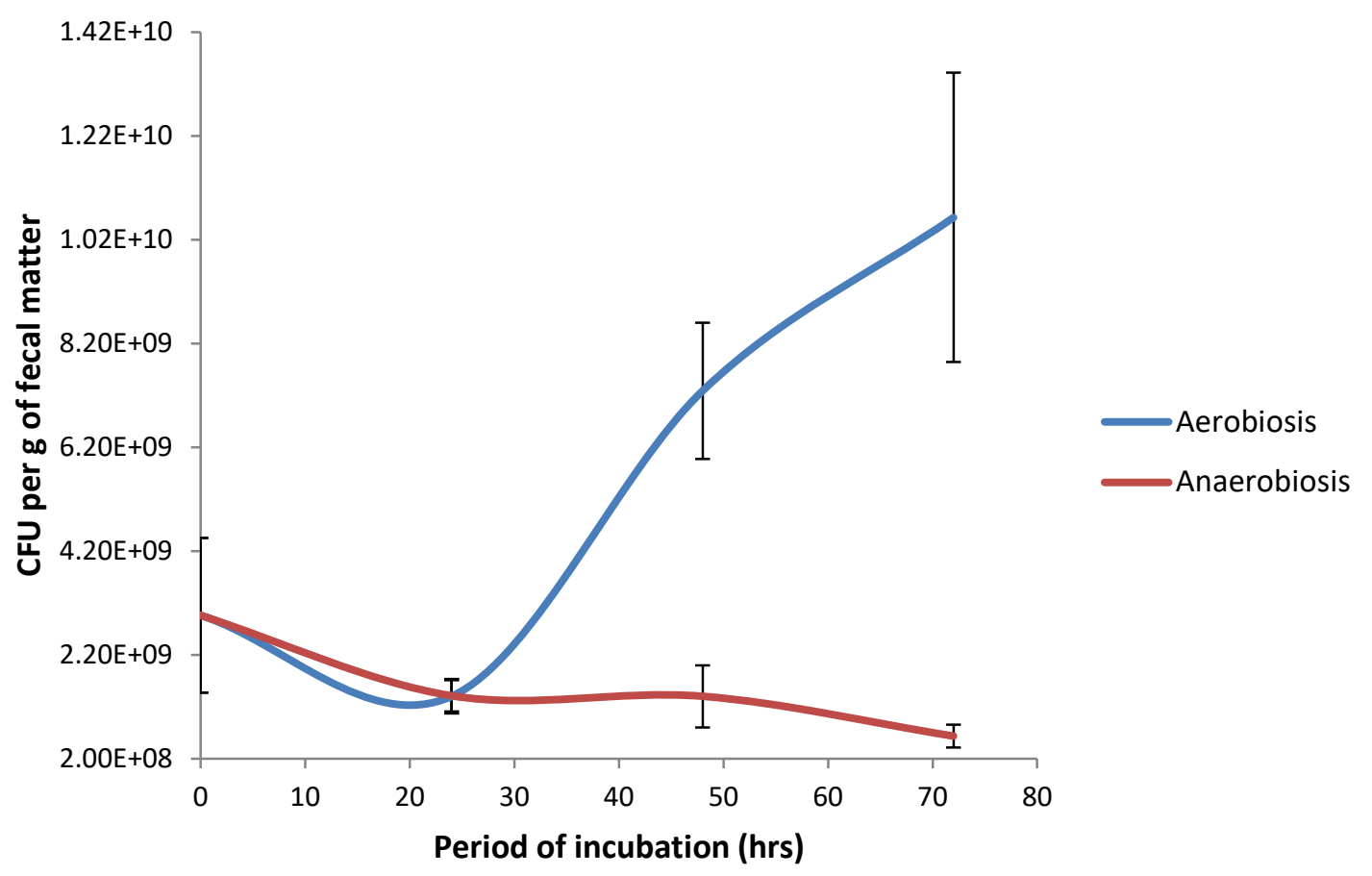

Fig. 3. Growth curves of Salmonella spp. in chicken fecal matter, under aerobic and anaerobic conditions. Curves were obtained for five Salmonella serovars (Infantis, Dublin, Heidelberg, Brandenburg, and Stanley) by $3 \mathrm{M}^{\mathrm{TM}}$ Molecular Detection Assay 2 - Salmonella (MDA2SAL). Bars indicate standard error.

The specific growth rate $(\mu)$ under aerobic conditions showed that during the first $48 \mathrm{hrs} E$. coli had the highest value decreasing during the following $24 \mathrm{hrs,}$ unlike Salmonella which started fast growth at this time (Fig. 4). We found that both Salmonella and E. coli multiplied in fecal matter outside a host. Our results indicate that Salmonella (as other Enterobacteriaceae) multiplies aerobically in fresh fecal matter (at higher levels than in the intestine) which may be a key step in the infective cycle. Our results show clear evidence that the fecal matter is a transient but very important component of the Enterobacteriaceae life cycle, where enterobacterial population expands (Russell and Jarvis, 2001; Vasco et al., 2015; Barrera et al., 2018), increasing the chances of reaching other hosts. Our study has an important limitation since we were unable to measure oxygen in fecal matter to confirm our results. 


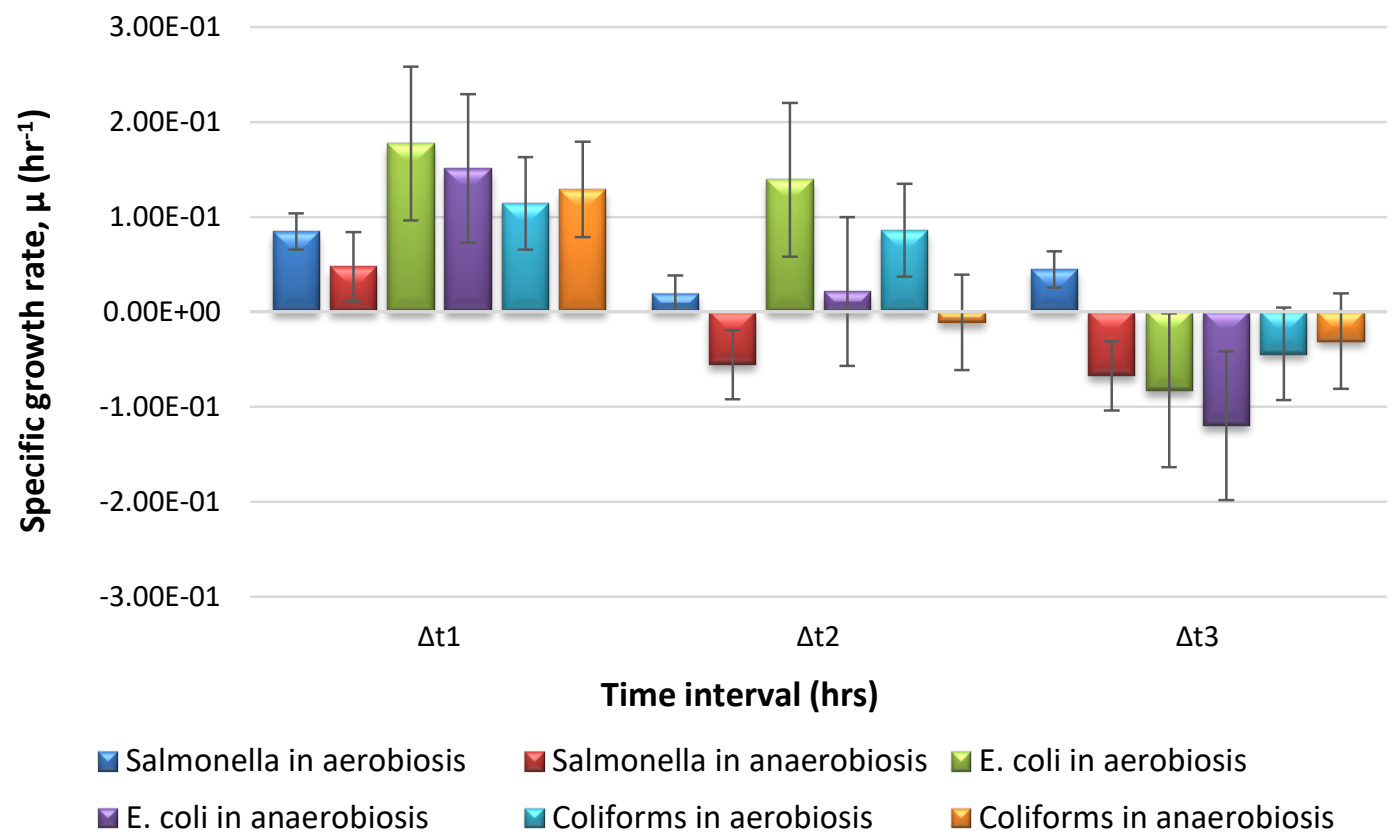

Fig. 4. Specific growth rate for Salmonella, E. coli and coliforms, under aerobic and anaerobic conditions. Specific growth rate, $\mu$, was calculated with the formula: $\mu=\frac{2.3 \log \left(\frac{\mathrm{N}}{\mathrm{No}}\right)}{\Delta \mathrm{t}}$, where $\mathrm{N}$ is the final population after a time interval of incubation, $\Delta t$, and No is the initial population. The incubation times were: $\mathrm{t} 1=0 \mathrm{hrs}, \mathrm{t} 2=24 \mathrm{hrs}, \mathrm{t} 3=48 \mathrm{hrs}$ and $\mathrm{t} 4=72 \mathrm{hrs}$. And the intervals were: $\Delta \mathrm{t} 1=\mathrm{t} 2-\mathrm{t} 1, \Delta \mathrm{t} 2=\mathrm{t} 3-\mathrm{t} 2$ and $\Delta \mathrm{t} 3=\mathrm{t} 4-\mathrm{t} 3$. Bars indicate standard error.

Microbiologists have struggled to explain why bacteria adapted to the anaerobic intestinal milieu possess energetically costly machinery to use oxygen (Govantes et al., 2000). Further, it has been shown that aerobic respiration is not important for Salmonella intestinal colonization (Barrow et al., 2015). We hypothesize that the reason for this apparent evolutionary mystery may be related to the enterobacterial ability to grow in fecal matter under aerobic conditions. Enterobacteriaceae are facultative anaerobic which can synthesize ATP by different enzymatic pathways, depending on the external concentration of $\mathrm{O}_{2}$ and the redox changes in the environment. When $\mathrm{O}_{2}$ is available, the bacteria obtain energy by aerobic respiration, with $\mathrm{O}_{2}$ being the final acceptor of electrons. In shortage of $\mathrm{O}_{2}$, these bacteria generate ATP by one of the following mechanisms: i) synthesis of terminal oxidases that allow the bacteria to take advantage of traces of $\mathrm{O}_{2} ; \mathrm{ii}$ ) use of other inorganic molecules (such as $\mathrm{NO}_{3}{ }^{-}$and $\mathrm{S}_{4} \mathrm{O}_{6}{ }^{2-}$ ) as final electron acceptors (Yamamoto and Droffner, 1985; Bueno et al., 2012; Rivera-Chávez et al., 
2013); iii) use of organic compounds as donors and acceptors (Madigan et al., 2012). Aerobic respiration produces much better performance in terms of ATP molecules per substrate molecule (Madigan et al., 2012).

Our results also have critical implications in waste management as we show that aerobic treatments may not be the best to reduce the numbers of Salmonella cells (Bueno et al., 2012; Rivera-Chávez et al., 2013). Additionally, the loose consistency of avian feces allows the entry of air and this phenomenon may contribute to the proficiency of these animals to spread Salmonella. Similarly, loose stools, caused by Salmonella infection, may favor the growth of this bacterium in fecal matter from animals with different fecal consistency.

Growth curves of Salmonella and E. coli at different incubation times suggest a negative correlation which may indicate that both species are competing possibly for oxygen, as was described in the intestine (Barrow et al., 2015; Velazquez et al., 2019). We speculate that, since E. coli multiplies initially more than Salmonella, it limits the growth of Salmonella until it decreases its growth rate. Once the growth of $E$. coli begins to decrease, Salmonella no longer must compete for oxygen and grows faster. Our findings disagree with the notion that once Salmonella and E. coli are excreted from the host, they enter a viable but not culturable status (Winfield and Groisman, 2003).

\section{References}

Barrera, S., Cardenas, P., Graham, J. P., and Trueba, G. (2018). Changes in dominant Escherichia coli and antimicrobial resistance after $24 \mathrm{hr}$ in fecal matter. MicrobiologyOpen, 8:e643. doi:10.1002/mbo3.643

Barrow, P. A., Berchieri, A., Freitas Neto, O. C. de, and Lovell, M. (2015). The contribution of aerobic and anaerobic respiration to intestinal colonization and virulence for Salmonella typhimuriumin in the chicken. Avian Pathology, 44(5), 401- 407. 
Bueno, E., Mesa, S., Bedmar, E. J., Richardson, D. J., and Delgado, M. J. (2012). Bacterial Adaptation of Respiration from Oxic to Microoxic and Anoxic Conditions: Redox Control. Antioxidants \& Redox Signaling, 16(8), 819-852. doi:10.1089/ars.2011.4051

Callejón, R. M., Rodríguez-Naranjo, M. I., Ubeda, C., Hornedo-Ortega, R., Garcia-Parrilla, M. C., and Troncoso, A. M. (2015). Reported Foodborne Outbreaks Due to Fresh Produce in the United States and European Union: Trends and Causes. Foodborne Pathogens and Disease, 12(1), 32-38. doi:10.1089/fpd.2014.1821

Gandelman, O. A., Church, V. L., Moore, C. A., Kiddle, G., Carne, C. A., Parmar, S., et al. (2010). Novel Bioluminescent Quantitative Detection of Nucleic Acid Amplification in RealTime. PLoS ONE, 5(11), e14155. doi:10.1371/journal.pone.0014155

Govantes, F., Albrecht, J. A., and Gunsalus, R. P. (2000). Oxygen regulation of the Escherichia coli cytochrome d oxidase (cydAB) operon: roles of multiple promoters and the Fnr-1 and Fnr-2 binding sites. Molecular Microbiology, 37(6), 1456-1469. doi:10.1046/j.1365-

2958.2000.02100.x

Khalid, S., Shahid, M., Natasha, Bibi, I., Sarwar, T., Haidair, A., and Niazi, N. (2018). A Review of Environmental Contamination and Health Risk Assessment of Wastewater Use for Crop Irrigation with a Focus on Low and High-Income Countries. International Journal of Environmental Research and Public Health, 15(5), 895. doi:10.3390/ijerph15050895

Kim, S., Frye, J. G., Hu, J., Fedorka-Cray, P. J., Gautom, R., and Boyle, D. S. (2006). Multiplex PCR-based method for identification of common clinical serotypes of Salmonella enterica subsp. enterica. Journal of Clinical Microbiology, 44(10), 3608-3615. doi.org/10.1128/JCM.00701-06 Madigan, M., Martinko, J., Stahl, D., and Clark, D. (2012). Brock Biology of Microorganisms (13th edn.). San Francisco, CA: Pearson Education, Inc. 
Maier, R. M. (2009). Bacterial Growth. In Environmental Microbiology, 37-54. Academic Press.

Inc. doi.org/10.1016/B978-0-12-370519-8.00003-1

Mandrell, R. E. (2009). Enteric Human Pathogens Associated with Fresh Produce: Sources, Transport, and Ecology. In Microbial Safety of Fresh Produce, 1-41. lowa, USA: Blackwell Publishing and the Institute of Food Technologists. doi:10.1002/9781444319347.ch1

Medrano-Félix, J. A., Castro-del Campo, N., Peraza Garay, F. de J., Martínez-Rodríguez, C. I., and Chaidez, C. (2017). Carbon source utilization-based metabolic activity of Salmonella Oranienburg and Salmonella Saintpaul in river water. Water and Environment Journal, 32(1), 118-124. doi:10.1111/wej.12306

Montville, T., Matthews, K., and Kniel, K. (2012). Food Microbiology. An Introduction (3rd. edn). Washington, DC: ASM Press. doi.org/10.1201/9781420027976.sec1

Rivera-Chávez, F., Winter, S. E., Lopez, C. A., Xavier, M. N., Winter, M. G., Nuccio, S.-P., et al. (2013). Salmonella Uses Energy Taxis to Benefit from Intestinal Inflammation. PLoS Pathogens, 9(4), e1003267. doi:10.1371/journal.ppat.1003267

Russell, J. B., and Jarvis, G. N. (2001). Practical mechanisms for interrupting the oral-fecal lifecycle of Escherichia coli. Journal of Molecular Microbiology and Biotechnology, 3(2), 265272.

Sandegren, L., Lindqvist, A., Kahlmeter, G., and Andersson, D. I. (2008). Nitrofurantoin resistance mechanism and fitness cost in Escherichia coli. Journal of Antimicrobial Chemotherapy, 62(3), 495-503. doi.org/10.1093/jac/dkn222

Sharma, M., Millner, P. D., Hashem, F., Vinyard, B. T., East, C. L., Handy, E. T., et al. (2019). Survival of Escherichia coli in manure-amended soils is affected by spatiotemporal, agricultural, and weather factors in the Mid-Atlantic United States. Appl Environ Microbiol, 85(5), 1-23. 
Reduce Nutrient Pollution Prior to Soil Application. Current Pollution Reports, 1(1), 47-

56. doi:10.1007/s40726-015-0005-1

Tiquia, S. M., Tam, N. F. Y., and Hodgkiss, I. J. (1998). Salmonella elimination during composting of spent pig litter. Bioresource Technology, 63(2), 193-196. doi:10.1016/s0960-

8524(97)00113-2

Vasco, G., Spindel, T., Carrera, S., Grigg, A., and Trueba, G. (2015). The role of aerobic respiration in the life cycle of Escherichia coli: Public health implications. Avances En Ciencias e Ingenierías, 7(2), B7-B9.

Velazquez, E. M., Nguyen, H., Heasley, K. T., Saechao, C. H., Gil, L. M., Rogers, A. W. L., et al. (2019). Endogenous Enterobacteriaceae underlie variation in susceptibility to Salmonella infection. Nature Microbiology, 4, 1057-1064. doi:10.1038/s41564-019-0407-8

Winfield, M. D., and Groisman, E. A. (2003). Role of Nonhost Environments in the Lifestyles of Salmonella and Escherichia coli. Applied and Environmental Microbiology, 69(7), 36873694. doi:10.1128/aem.69.7.3687-3694.2003

Yamamoto, N., and Droffner, M. L. (1985). Mechanisms determining aerobic or anaerobic growth in the facultative anaerobe Salmonella typhimurium. Proc. Natl. Acad. Sci. USA, 82(April), 2077-2081.

\section{Conflict of interest}

The authors declare no conflict of interest.

\section{Supporting Information}


bioRxiv preprint doi: https://doi org/10.1101/766782; this version posted September 12 2019. The copyright holder has placed this preprint (which was not certified by peer review) in the Public Domain. It is no longer restricted by copyright. Anyone can legally share, reuse, remix, or adapt this material for any purpose without crediting the original authors.

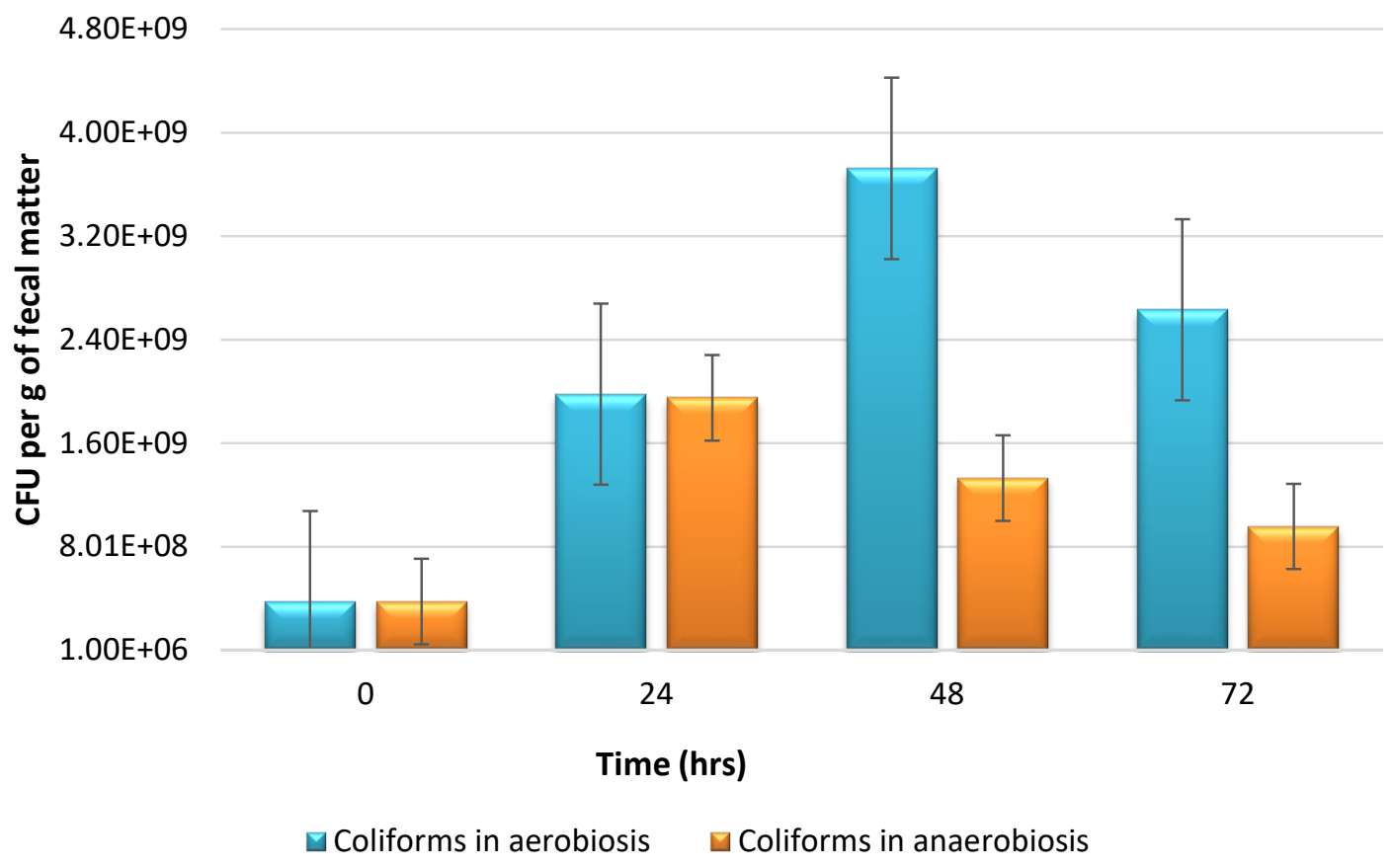

Fig. S1. Growth of coliforms in chicken fecal matter, under aerobic and anaerobic conditions. The number of coliforms corresponded to the sum of the red and blue colonies with gas in $3 \mathrm{M}^{\mathrm{TM}}$ Petrifilm E. coli/Coliform Count Plates incubated 24 and $48 \mathrm{hrs}$. Bars indicate standard error. 